\title{
Minimally invasive endoscopic-assisted posterior thoracic sympathectomy
}

\author{
Paul A. Gardner, M.D., Pawel G. Ochalski, M.D., and John J. Moossy, M.D. \\ Department of Neurological Surgery, University of Pittsburgh Medical Center, \\ Pittsburgh, Pennsylvania
}

\begin{abstract}
Palmar hyperhidrosis is a disorder of the autonomic nervous system characterized by excessive perspiration of the palms, but may involve other body parts as well. Traditional posterior approaches have been performed less often in favor of less invasive thoracoscopic sympathectomies, which have a high success rate with low associated morbidity. However, some patients are not candidates for a transthoracic surgery and may need an alternative treatment strategy.

In situations in which a posterior approach may be necessary, the authors have developed a minimal access endoscopic-assisted dorsal sympathectomy procedure, applying minimally invasive spine muscle splitting techniques. The authors believe that the development of this technique may help to minimize surgical morbidity associated with the traditional posterior approach by reducing pain, tissue damage, and length of postoperative recovery. This paper is a report on the successful treatment of palmar hyperhidrosis using a minimally invasive posterior technique and describes the surgical approach and outcomes in 2 patients who have been treated in this manner.

Two patients underwent minimally invasive endoscopic-assisted posterior thoracic sympathectomy for hyperhidrosis. Both patients experienced relief of their symptoms after surgery with follow-up durations of 32 and 9 months and length of stays of 0.9 and 2.8 days, respectively. One patient suffered a unilateral Horner syndrome and underwent an eyelid lift. The other patient was readmitted to the hospital 2 days after discharge with atelectasis. She was obese and suffered from chronic obstructive pulmonary disease at baseline, which were reasons she opted for a posterior approach. Neither patient suffered a pneumo- or hemothorax.

Minimally invasive endoscopic-assisted posterior thoracic sympathectomy can be safely performed for relief of hyperhidrosis. The procedure has risks for the usual complications of sympathectomy. This technique may provide an alternative to thoracoscopic approaches, especially in those patients with pulmonary disease or obesity.
\end{abstract} (DOI: $10.3171 /$ FOC/2008/25/8/E6)

\section{KEY WORDS • endoscope • hyperhidrosis • minimally invasive surgery • posterior thoracic sympathectomy}

$\mathrm{P}$ ALMAR hyperhidrosis is an idiopathic disorder characterized by excessive perspiration of the palms in excess of usual physiological needs. It most commonly affects the palms but can involve the feet, axillae, face, and scalp with different combinations of severities and locations. Hyperhidrosis may be secondary to a variety of central nervous system lesions or a manifestation of systemic disease such as hyperthyroidism or pheochormocytoma. ${ }^{2,12}$ More commonly, it is idiopathic (primary or essential) with an incidence rate ranging from 0.15 to $1 \%$ and may be even higher in certain populations. ${ }^{1,3}$ The pathophysiology of primary palmar hyperhidrosis is believed to be hyperactivity of the sympathetic fibers that pass through the upper sympathetic chain at the T-2 and T-3 levels, ${ }^{20}$ resulting in increased eccrine gland sweat production. Hyperhidrosis can be very distressing for patients and significantly affects their quality of life, especially when it limits their social and professional activities.

Abbreviations used in this paper: $\mathrm{AP}=$ anteroposterior; $\mathrm{BMI}=$ body mass index.
The treatment options for primary hyperhidrosis include a wide range of medical and nonsurgical techniques as well as psychological therapy. ${ }^{5}$ However, when these conservative measures fail and the condition remains intractable, then surgical intervention is indicated. In the last 100 years, several surgical approaches have been used to treat hyperhidrosis including axillary gland excision, subcutaneous curettage of the axillae, and surgical sympathectomy. For many years, open sympathectomy had been the approach used to treat this condition, with the first such procedure performed in $1920 .{ }^{10}$ Since then, several patient series have been published concerning open approaches using thoracotomy, supraclavicular, axillary, anterior, or posterior paraspinal exposures with resolution of symptoms in $>$ $95 \%$ of patients in some series..$^{1,4,15}$

More recently, however, video-assisted thoracoscopic sympathectomy has replaced open approaches as the standard technique. ${ }^{6,13,18}$ High success rates have been reported with a reduction in the complications that are associated with traditional open approaches such as brachial plexopathy, Horner syndrome, and chylothorax. Since the first 


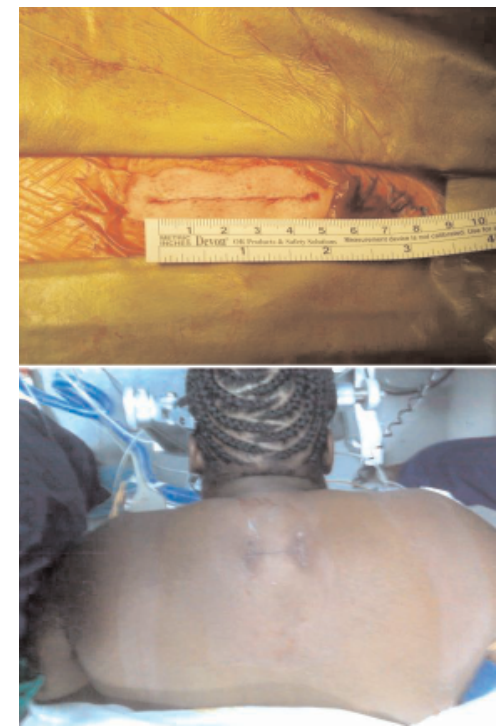

FIG. 1. Intraoperative photographs of the incisions used in the minimally invasive procedure. Upper: Photograph of a single midline incision used for a minimally invasive posterior approach for sympathectomy. The skin incision was generous and pulled laterally to accommodate separate fascial incisions. A larger than necessary incision was used because this was the first procedure performed and a generous incision was made to limit any difficulties related to the incision. Lower: Photograph of bilateral minimally invasive endoscopic-assisted posterior thoracic sympathectomy incisions. No measuring tool was photographed, but the incisions were $\sim 3 \mathrm{~cm}$.

report on using thoracoscopy to perform a sympathectomy by Hughes in $1942,{ }^{9}$ followed by Kux's ${ }^{11}$ experience in more than 1400 patients using endoscopic thoracic sympathectomy, this approach has become the method of choice for many surgeons. The advantages of performing thoracoscopic sympathectomy under video guidance relate to improved visualization, which maximizes surgical safety without reducing efficacy. ${ }^{7,21}$ Nevertheless, thoracoscopic sympathectomy requires 2 separate positionings during the procedure to access each sympathetic chain and the ipsilateral lung must be deflated one at a time. As a result, the thoracoscopic approach can be complicated by the development of a hemothorax and pneumothorax, with an incidence rate ranging from 1 to $10 \% .^{3,8,18}$ Thus, this proce-

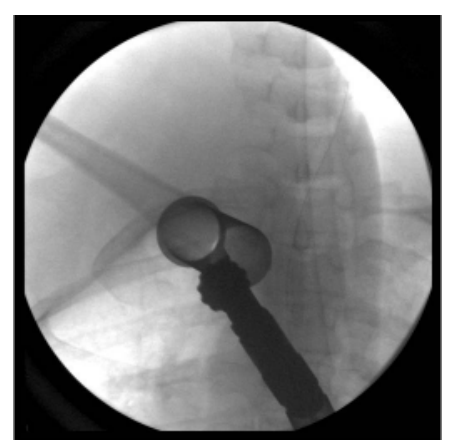

FIG. 2. Intraoperative posteroanterior fluoroscopic view of a standard minimally invasive spine tubular retractor docked on the $\mathrm{T}-3$ costotransverse joint following sequential dilation.

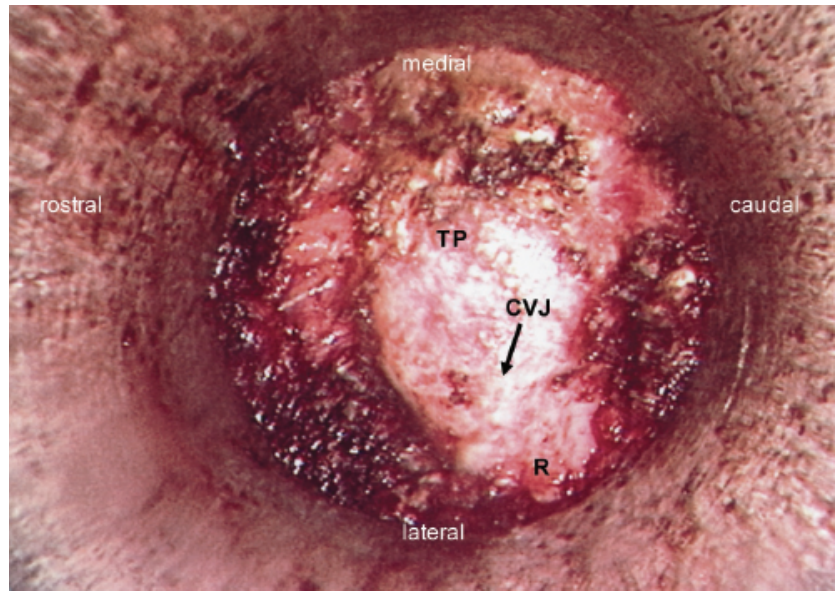

FIG. 3. Intraoperative endoscopic view through the minimally invasive spine tube showing the left $\mathrm{T}-3$ costotransverse junction $(\mathrm{CVJ}) . \mathrm{R}=$ third rib; $\mathrm{TP}=$ transverse process.

dure may not be safe for all patients, especially those who are morbidly obese or have severe pulmonary disease..$^{14}$

In this paper, we present our experience performing a dorsal approach for upper thoracic sympathectomy using minimally invasive muscle-splitting techniques adopted from spine procedures (tubular dilation for working port access ${ }^{16}$ ) and assisted by endoscopic visualization. We have been successful in treating 2 patients using this approach without any increased morbidity to the patients. This report focuses on describing the details of this technique and proposes guidelines for selecting the patients who may benefit the most from this approach.

\section{Surgical Technique}

The patient is placed prone on chest rolls, in head pins, on a radiolucent operating room table. Following standard surgical preparation of the patient, AP fluoroscopic localization of the T-3 costotransverse junction is performed and the incision is centered around this mark. Bilateral paramedian skin incisions or a single midline incision with bilateral fascial openings via lateral skin retraction is performed (Fig. 1). Sequential dilation over a K-wire overlying the costotransverse junction $(\sim 2-2.5 \mathrm{~cm}$ from midline) is performed under fluoroscopic guidance (Fig. 2). Precise localization is critical to avoid either a high sympathetic sec-

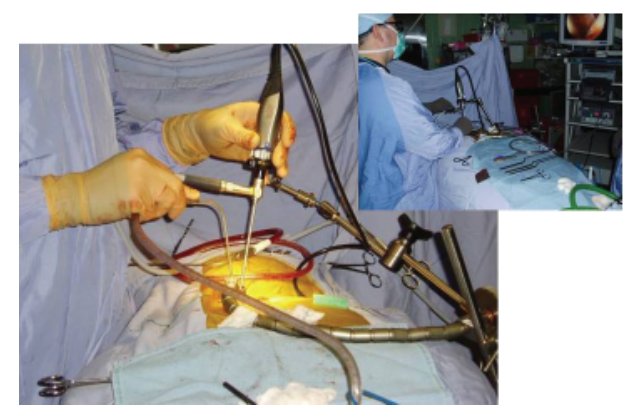

FIG. 4. Photographs of the operative setup showing a tubular retractor and endoscope on a holder. 


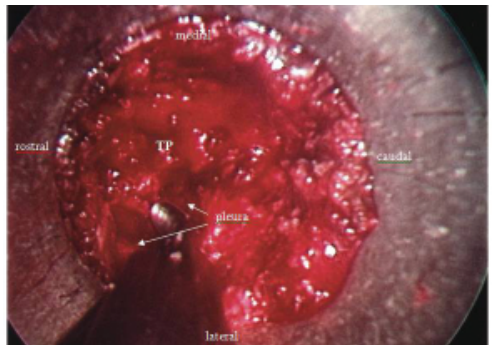

FIG. 5. Intraoperative endoscopic view of the removal of the T3 transverse process (TP) on the left. The underlying pleura must be carefully dissected from the transverse process to avoid the development of a pneumothorax.

tioning and resultant Horner syndrome or low sectioning with an inadequate result. Given the level involved, this localization must be performed using AP chest fluoroscopy, counting from the first rib down.

Fascial dilation is performed at a slight medial angle to allow for visualization of the sympathetic chain as it runs just under the transverse processes on the vertebral body. A tubular retractor is then docked on the costotransverse joint (Figs. 2 and 3). Under endoscopic or microscopic visualization (Fig. 4), the medial rib head and the transverse process are removed using a high-speed drill. The underlying pleura is carefully dissected away from the transverse process (Fig. 5). Sometimes the sympathetic chain falls away from the lateral vertebral body and can be easily located on the pleura; if not, an endoscope helps to visualize the lateral vertebral body and identify the sympathetic chain (Fig. 6). The ganglion is identified and hemoclip ligation and sectioning is performed superior and inferior to the ganglion (Fig. 7). Intraoperative pathological analysis can be helpful for confirming that the sympathetic ganglion was resected. In addition, final radiographic confirmation of hemoclip placement at the T-3 level is performed (Fig. 8). The pleura is inspected to ensure that there is no tear. Finally, sterile irrigation is used to partially fill the tube and immerse the pleura to inspect it for an air leak. If an air leak is noted, a red rubber or pigtail catheter can be placed in the pleural space and tunneled percutaneously to ensure that there is no postoperative pneumothorax. The entire process is repeated on the contralateral side (Fig. 9).

\section{Results}

We performed thoracic sympathectomies for hyperhidrosis via a minimally invasive posterior approach in 2 patients (Table 1). Both patients were young females who requested posterior approaches, our standard approach. The second patient had chronic obstructive pulmonary disease

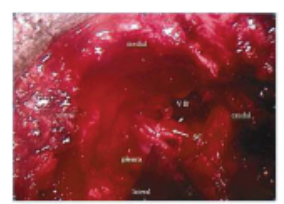

FIG. 6. Intraoperative endoscopic view of the sympathetic chain (SC) looped over a nerve hook after dissection away from the lateral vertebral body (VB)

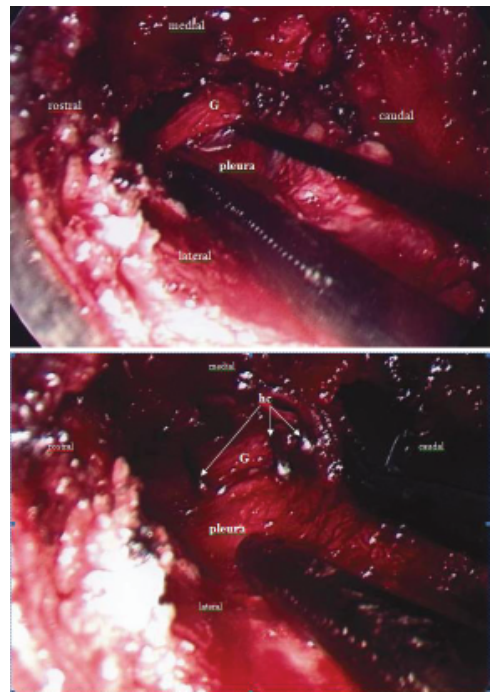

FIG. 7. Intraoperative endoscopic views of the T-2 sympathetic ganglion undergoing clip ligation. Upper: View showing the T-2 ganglion $(\mathrm{G})$ dissected and separated for clip ligation. Lower: View showing hemoclips (hc) superior and inferior to the T-2 ganglion prior to ligation.

and obesity (BMI 32.5) as potential complicating factors for a thoracoscopic approach. Complications included a unilateral Horner syndrome that has not resolved since surgery in the first case and postoperative atelectasis without evidence of a pneumothorax in the second case. The Horner syndrome was believed to be a traction injury. The operative level was rechecked and confirmed on postoperative radiographs and the contralateral side (at the same level) did not result in Horner syndrome. Although complete resolution of the initial presenting area of hyperhidrosis resolved in both patients, the patient in Case 2 reported compensatory hyperhidrosis in the axillae. Both patients reported a significant improvement in their social and professional activities following the procedures with follow up of 32 and 9 months, respectively.

\section{Discussion}

Thoracoscopic sympathectomies for hyperhidrosis have largely replaced open sympathectomy procedures, and most of these thoracoscopic sympathectomies are performed by thoracic surgeons. However, in cases of significant pulmonary disease in which the thoracic cavity is ob-

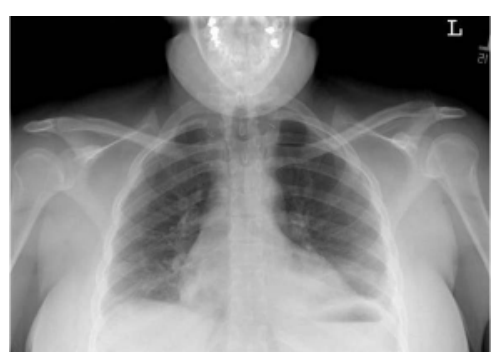

FIG. 8. Postoperative AP chest radiograph showing bilateral T3 costotransversectomies and hemoclips. 


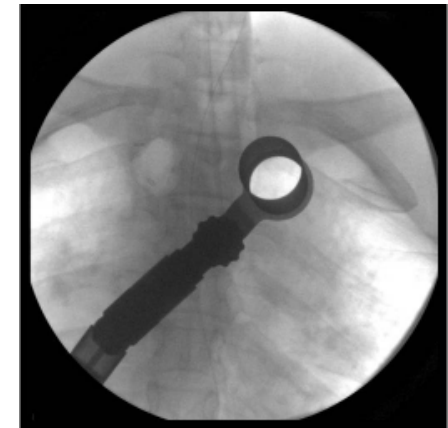

FIG. 9. Intraoperative posteroanterior radiograph showing the tube system docked over the contralateral T-3 costotransverse junction for completion of bilateral sympathectomies.

literated as well as in obese patients, a posterior open approach may be considered.

In the last decade a significant effort has been made toward minimizing surgical morbidity involved in many aspects of spine surgery. Newer techniques and instrumentation have enabled spine surgeons to perform a variety of traditional spinal surgeries through smaller incisions resulting in decreased muscle retraction with subsequent reduction in tissue injury. These advances may reduce the morbidity of postoperative pain as well as wound infections associated with traditional open approaches. ${ }^{16,19}$ By applying similar minimally invasive techniques, we have been able to successfully perform an open dorsal upper thoracic sympathectomy using minimally invasive spine musclesplitting techniques assisted by endoscopic visualization. We propose using this approach in situations in which a patient may not be a candidate for thoracoscopic sympathectomy or if a patient requests a posterior approach.

The fact that both procedures resulted in complications demonstrates the potential difficulties with this procedure and patient population. In the first patient, a Horner syndrome resulted, likely as a result of traction on the rostral ganglion. This complication may be a reflection of using a different technique, but it is also a known complication with standard techniques. The second patient suffered a pulmonary complication (likely as a result of her obesity and preexisting pulmonary disease) that was self-limited, easily managed, and without long-term consequence. This patient also developed some compensatory axillary hyperhidrosis, a known sequelae that was discussed with the patient preoperatively. She is satisfied with her operative result despite this.

The minimally invasive dorsal thoracic sympathectomy shares similar advantages with the standard open posterior approach as previously described by Cloward ${ }^{4}$ and by
Pillay et al. ${ }^{17}$ Both open and minimal-access approaches enable bilateral exposure of the sympathetic chains and ganglia at a single operation without the need to reposition the patient intraoperatively. This advantage avoids potential complications related to patient positioning such as pressure ulcers, dislodging of endotracheal tubes, and others. In addition, intentional entry into the pleural space (as is done in a thoracoscopic approach) is avoided while performing a posterior approach, thus potentially decreasing the incidence of a hemo- or pneumothorax. Furthermore, a minimal access approach can be easily incorporated into a surgeon's repertoire provided they feel comfortable with newer minimally invasive spine techniques, similar to the advantage the open posterior approach provides for the neurosurgeon because it incorporates an exposure similar to that of a thoracic laminectomy. Differences between the minimal access sympathectomy and the traditional open approach begin with a smaller incision. By using minimally invasive spine muscle-splitting techniques, tissue damage is limited, which may help decrease postoperative pain and decrease hospital stays when compared with traditional posterior approaches. Also, the addition of endoscopic assistance improves visualization and localization of the sympathetic chain, often one of the most difficult and timeconsuming parts of the posterior approach.

\section{Conclusions}

We propose using a minimal access technique in patients who require a posterior approach for upper thoracic sympathectomies. From our case experience we believe that this procedure is a safe and effective management strategy for palmar and axillary hyperhidrosis in selected patients. Further expansion of this case series will be necessary to better evaluate long-term outcomes.

\section{References}

The authors do not report any conflict of interest concerning the materials or methods used in this study or the findings specified in this paper.

\section{References}

1. Adar R, Kurchin A, Zweig A, Mozes M: Palmar hyperhidrosis and its surgical treatment: a report of 100 cases. Ann Surg 186:34-41, 1977

2. Awada A, Ammar A, al-Rajeh S, Borollosi M: Excessive sweating: an uncommon sign of basilar artery occlusion. J Neurol Neurosurg Psychiatry 54:277-278, 1991

3. Chiou TS, Chen SC: Intermediate-term results of endoscopic transaxillary T2 sympathectomy for primary palmar hyperhidrosis. Br J Surg 86:45-47, 1999

4. Cloward RB: Hyperhydrosis. J Neurosurg 30:545-551, 1969

TABLE 1

Summary of the 2 patients treated using minimally invasive endoscopic-assisted posterior thoracic sympathectomies*

\begin{tabular}{|c|c|c|c|c|c|c|c|}
\hline $\begin{array}{l}\text { Case } \\
\text { No. }(\end{array}$ & $\begin{array}{l}\text { Age } \\
\text { yrs), Sex }\end{array}$ & $\begin{array}{l}\text { Hyperhi- } \\
\text { drosis Type }\end{array}$ & $\begin{array}{l}\text { Follow- } \\
\text { up (mos) }\end{array}$ & $\mathrm{BMI}$ & $\begin{array}{l}\text { LOS } \\
\text { (days) }\end{array}$ & Complications & Effectiveness of Procedure \\
\hline 1 & $16, \mathrm{~F}$ & palmar, axillary & 32 & 21.0 & 0.9 & unilateral Horner syndrome & complete resolution \\
\hline 2 & $36, \mathrm{~F}$ & palmar, feet & 9 & 32.5 & 2.8 & $\begin{array}{l}\text { atelectasis } 1 \text { wk postop.; } \\
\text { no pneumothorax }\end{array}$ & $\begin{array}{l}\text { complete resolution of palmar and foot but } \\
\text { compensatory axillary hyperhidrosis }\end{array}$ \\
\hline
\end{tabular}

* LOS = length of stay. 
5. Connolly M, de Berker D: Management of primary hyperhidrosis: a summary of the different treatment modalities. Am J Clin Dermatol 4:681-697, 2003

6. Han PP, Gottfried ON, Kenny KJ, Dickman CA: Biportal thoracoscopic sympathectomy: surgical techniques and clinical results for the treatment of hyperhidrosis. Neurosurgery 50:306-312, 2002

7. Hashmonai M, Kopelman D, Schein M: Thoracoscopic versus open supraclavicular upper dorsal sympathectomy: a prospective randomised trial. Eur J Surg Suppl 572:13-16, 1994

8. Herbst F, Plas EG, Függer R, Fritsch A: Endoscopic thoracic sympathectomy for primary hyperhidrosis of the upper limbs. A critical analysis and long-term results of 480 operations. Ann Surg 220:86-90, 1994

9. Hughes J: Endoscopic sympathectomy. Proc R Soc Med 35: $585-586,1942$

10. Kotzareff A: Résection partielle du tronc sympathique cervical droit pour hyperhidrose unilatérale (regions faciale, cervicale, thoracique et brachiale droites). Rev Med Suisse Romande 40:111113,1920

11. Kux E: The endoscopic approach to the vegetative nervous system and its therapeutic possibilities; especially in duodenal ulcer, angina pectoris, hypertension and diabetes. Dis Chest 20:139-147, 1951

12. Labar DR, Mohr JP, Nichols FT III, Tatemichi TK: Unilateral hyperhidrosis after cerebral infarction. Neurology 38:1679-1682, 1988

13. Lin TS, Fang HY: Transthoracic endoscopic sympathectomy in the treatment of palmar hyperhidrosis - with emphasis on perioperative management (1,360 case analyses). Surg Neurol 52:453457, 1999

14. Noppen M, Vincken W: Thoracoscopic sympathicolysis for essen- tial hyperhidrosis: effects on pulmonary function. Eur Respir J 9:1660-1664, 1996

15. Palumbo LT: Anterior transthoracic approach for upper thoracic sympathectomy. AMA Arch Surg 72:659-666, 1956

16. Perez-Cruet MJ, Foley KT, Isaacs RE, Rice-Wyllie L, Wellington R, Smith MM, et al: Microendoscopic lumbar discectomy: technical note. Neurosurgery 51 (5 Suppl): S129-S136, 2002

17. Pillay PK, Awad IA, Dohn DF: Upper thoracic sympathectomy by a posterior midline approach, in Rengachary SS, Wilkins RH (eds): Neurosurgical Operative Atlas. Park Ridge, IL: Williams \& Wilkins, 1991, pp 267-273

18. Rex LO, Drott C, Claes G, Gothberg G, Dalman P: The Borås experience of endoscopic thoracic sympathicotomy for palmar, axillary, facial hyperhidrosis and facial blushing. Eur J Surg Suppl 580:23-26, 1998

19. Schizas C, Tsiridis E, Saksena J: Microendoscopic discectomy compared with standard microsurgical discectomy for treatment of uncontained or large contained disc herniations. Neurosurgery 57 (4 Suppl):357-360, 2005

20. Shih CJ, Wu JJ, Lin MT: Autonomic dysfunction in palmar hyperhidrosis. J Auton Nerv Syst 8:33-43, 1983

21. Zacherl J, Imhof M, Huber ER, Plas EG, Herbst F, Jakesz R, et al: Video assistance reduces complication rate of thoracoscopic sympathicotomy for hyperhidrosis. Ann Thorac Surg 68:11771181, 1999

Manuscript submitted May 15, 2008.

Accepted May 30, 2008.

Address correspondence to: Paul A. Gardner, M.D., UPMC Presbyterian Hospital, Department of Neurological Surgery, 200 Lothrop Street, Suite B-400, Pittsburgh, Pennsylvania 15213. email: gardpa@upmc.edu. 